\title{
Utilização do leito de jorro para a aplicação de revestimento entérico em cápsulas gelatinosas duras
}

\author{
Gilmarcio Zimmermann Martins, Wanderley Pereira de Oliveira*
}

Departamento de Ciências Farmacêuticas, Faculdade de Ciências Farmacêuticas de Ribeirão Preto, Universidade de São Paulo

*Correspondência:

W. P. Oliveira

Departamento de Ciências

Farmacêuticas

FCFRP-USP

Via do Café, S/N - Bairro Monte Alegre 14040-903 - Ribeirão Preto - SP - Brasil

E-mail: wpoliv@fcfrp.usp.br
Apresenta-se nesse trabalho um estudo da viabilidade da aplicação de revestimento gastro-resistente em cápsulas gelatinosas duras pelo processo leito de jorro. As variáveis investigadas foram: a razão entre a vazão mássica de suspensão de revestimento alimentada pela vazão de gás introduzida ao sistema $\left(W_{s} / W_{g}\right)$, a razão entre a vazão de ar de jorro pela vazão correspondente à condição de jorro mínimo $\left(Q / Q_{j m}\right)$, massa inicial de cápsulas inseridas no equipamento $\left(M_{0}\right)$ e o tamanho das cápsulas $\left(T_{\text {caps }}\right)$. $O$ processo e a qualidade do produto obtido foram analisados através da taxa de aumento de massa das cápsulas $\left(K_{p}\right)$, da eficiência de adesão durante a operação ( $\eta$ ), ensaios de desintegração, ensaios de compressão e por microfotografias obtidas em microscópio eletrônico de varredura. Os experimentos foram realizados em um leito de jorro com coluna cilindrica de $15 \mathrm{~cm}$ de diâmetro e base cônica com ângulo incluso de $40^{\circ}$. A análise dos resultados obtidos levou à determinação da melhor condição para a aplicação de revestimento de cápsulas: $W_{s} / W_{g}=0,0014$ e $Q / Q_{j m}=3,0$, para ambas as massas de cápsulas introduzidas no sistema e tamanhos de cápsulas estudados. Os ensaios de desintegração mostraram que massas de revestimento superiores a $0,006 \mathrm{~g} / \mathrm{cm}^{2}$ proporcionam o efeito gastro-resistente.
Unitermos:

- Leito de jorro

- Revestimento entérico

- Eudragit L-30D-55

- Cápsulas gelatinosas duras

\section{INTRODUÇÃO}

As cápsulas gelatinosas duras têm sofrido significativo aumento na sua comercialização e, atualmente, essa forma farmacêutica possui grande aceitação, constituindo, ao lado dos comprimidos, as formas farmacêuticas mais utilizadas de administração oral de medicamentos (Pina et al., 1996, 1997). Esse crescimento na comercialização deve-se principalmente às vantagens de manipulação e administração atribuídas a esse produto.

Entretanto, algumas desvantagens são associadas a esta forma farmacêutica, que incluem a impossibilidade de utilização de drogas que se oxidam e/ou que são degradadas ou inativadas em meio ácido, como por exemplo, hormônios, peptídeos e enzimas. Existem situações em que o fármaco pode irritar a mucosa gástrica, causando ná- 
useas, vômitos ou até mesmo ulcerações quando administrado por via oral. Em outros casos, a substância medicamentosa só possui eficácia quando liberada em determinada região do intestino, seja ele o duodeno, jejuno, ílio ou intestino grosso (Bodmeier, 1999; Felton et al., 1995, 1996; Kalala et al., 1996; Cole et al., 2002).

Para que esses fármacos possam ser encapsulados é necessário alterar o perfil de liberação da cápsula gelatinosa dura, de modo que a desintegração da cápsula e a dissolução do fármaco ocorram somente em $\mathrm{pH}$ neutro e/ou básico (valores esses encontrados no meio entérico), o que pode ser conseguido com a aplicação de um revestimento gastroresistente. Entretanto, na literatura existem poucos estudos sobre a aplicação de revestimento nessas formas farmacêuticas. Portanto, o desenvolvimento de métodos para a aplicação de revestimento em cápsulas gelatinosas duras desponta como um promissor campo de pesquisas.

Por outro lado, dentre os vários tipos de processos existentes atualmente para se realizar operações de revestimento em partículas, o processo leito de jorro tem se demonstrado promissor devido à facilidade de operação, controle e pela qualidade do produto obtido. Esse processo tem sido amplamente empregado para a aplicação de revestimento em produtos químicos, agrícolas e farmacêuticos, tais como sementes, agrotóxicos, fertilizantes, compostos nucleares e comprimidos (Porter, 1978; Doane et al., 1977; Salman, 1989; Salman et al., 1989; Piccinini, Rovero, 1983; Oliveira, 2002). Em particular, tem sido atribuída a esse processo a vantagem de formação de camada altamente uniforme em curto tempo de processamento, devido, principalmente, às excelentes condições de transferência de calor e de massa no interior do equipamento (Kucharski, Kmiec, 1983).

Em face disso, foi desenvolvido este trabalho que visa avaliar a utilização do leito de jorro para a aplicação de revestimento gastro-resistente em cápsulas gelatinosas duras, analisando-se a influência de vários parâmetros operacionais sobre o desempenho do processo e sobre a qualidade do produto obtido.

\section{MATERIAL E MÉTODOS}

\section{Material}

A composição de revestimento utilizada foi composta de $15,00 \%$ de Eudragit ${ }^{\circledR}$ L30 D55, 3,00\% de trietil citrato (plastificante), $0,15 \%$ de carboximetilcelulose, $4,85 \%$ talco Farmacêutico e 77,00 \% de água destilada (em peso).

Como suporte para a aplicação do revestimento foram empregadas cápsulas duras de gelatina com duas di- ferentes dimensões (números 0 e 1), preenchidas com lactose. Essas cápsulas foram caracterizadas através da determinação de sua massa média unitária, $\mathrm{m}_{\mathrm{p} 0}$, do diâmetro médio volumétrico, $\mathrm{d}_{\mathrm{p}}$; da superfície específica, Se e do fator de forma, $\phi$. O procedimento adotado para a determinação dessas propriedades está apresentado em Oliveira (2002). A Tabela I apresenta os resultados dessa caracterização.

TABELA I - Caracterização física das cápsulas gelatinosas duras utilizadas

\begin{tabular}{ccccc}
\hline $\begin{array}{c}\mathbf{T}_{\text {caps }} \\
\mathbf{n}^{\mathbf{0}}\end{array}$ & $\begin{array}{c}\mathbf{m}_{\mathbf{p 0}} \\
(\mathbf{g})\end{array}$ & $\begin{array}{c}\mathbf{d}_{\mathbf{p}} \\
(\mathbf{c m})\end{array}$ & $\begin{array}{c}\mathbf{S e} \\
\left(\mathbf{c m}^{2} / \mathbf{g}\right)\end{array}$ & $\begin{array}{c}\boldsymbol{\phi} \\
(-)\end{array}$ \\
\hline 0 & 0,47 & 1,04 & 5,13 & 0,86 \\
1 & 0,35 & 0,93 & 5,50 & 0,86 \\
\hline
\end{tabular}

\section{Equipamentos}

Os experimentos foram realizados em equipamento do tipo leito de jorro, com diâmetro de coluna de $15 \mathrm{~cm}$, base cônica com ângulo incluso de $40^{\circ}$ e diâmetro do orifício de entrada do gás de $3,3 \mathrm{~cm}$. Todas as peças foram construídas em aço-inox. O equipamento possui como acessórios um soprador (marca Ibram, de 7,5 HP de potência), medidor de vazão tipo placa de orifício, conectado a um manômetro diferencial tipo tubo em $U$, rotâmetro, para a medida da vazão do ar comprimido, sistema de alimentação da suspensão de revestimento e sistema de aquisição de dados fluidodinâmicos e térmicos. A Figura 1 apresenta diagrama esquemático do equipamento utilizado.

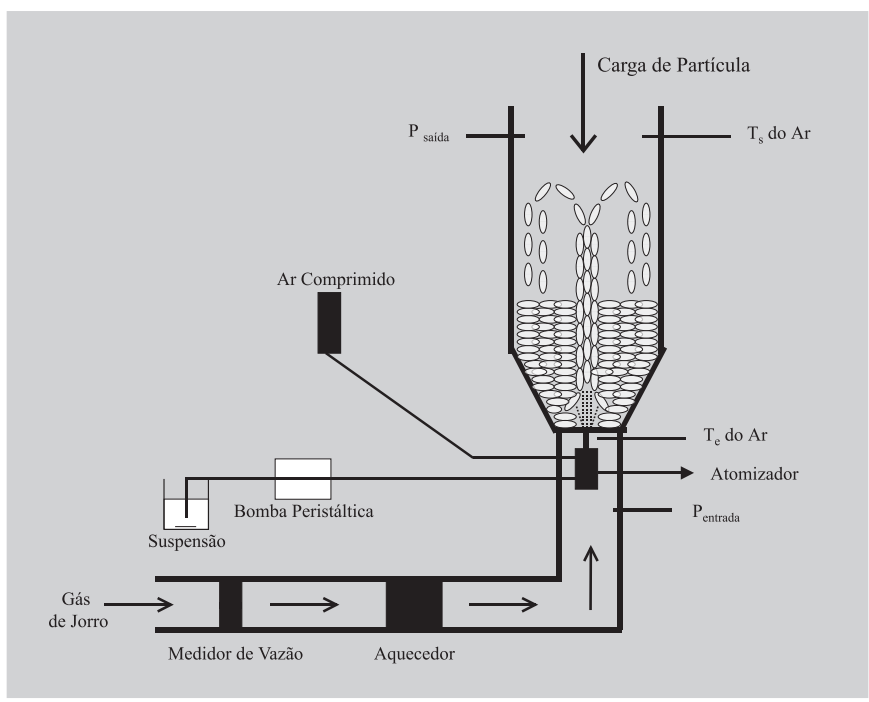

FIGURA 1 - Diagrama esquemático do equipamento utilizado. 


\section{Métodos experimentais}

\section{Caracterização fluidodinâmica do equipamento}

A fluidodinâmica do equipamento foi caracterizada por meio da determinação da velocidade de jorro mínimo, queda de pressão máxima no leito e queda de pressão de jorro estável. Esses parâmetros foram obtidos através da obtenção de gráficos de queda de pressão no equipamento em função da vazão de ar introduzida no sistema (Mathur, Epstein, 1974). Inicialmente, construiu-se uma curva de queda de pressão com o leito vazio, para a determinação da perda de carga no equipamento sem a presença de partículas inertes. A seguir, colocou-se no leito a massa pré-determinada de cápsulas gelatinosas duras $\left(\mathrm{M}_{0}\right.$ $=150$ e $300 \mathrm{~g}$ ) e injetou-se ar pelo orifício de entrada do leito, aumentando lentamente sua vazão, a qual é determinada por uma placa de orifício conectada a um transdutor de pressão e a um sistema de aquisição de dados, configurado para coletar 4 pontos por segundo. O valor correspondente à queda de pressão entre a entrada (parte inferior do orifício de entrada do gás) e a saída do leito foi determinada com o auxílio de outro transdutor de pressão. Atingido o jorro, iniciou-se o procedimento inverso, ou seja, diminuiu-se gradualmente a vazão de ar, medindo novamente a queda de pressão. Aos valores de queda de pressão assim determinados foram subtraídos os valores correspondentes a queda de pressão do leito vazio.

\section{Operação de revestimento}

A operação teve início com a introdução no equipamento de uma carga de partículas previamente pesadas. Estabelecido o jorro, realizou-se o aquecimento do ar até a temperatura de $60^{\circ} \mathrm{C}$. Atingida está temperatura, iniciou-se a alimentação da suspensão de revestimento e do ar de atomização. Amostras significativas de cápsulas foram retiradas do leito em intervalos de tempo regulares $(0,15,30$ e 45 minutos). A seguir, as amostras foram pesadas, permitindo a determinação dos valores experimentais da taxa de aumento de massa das partículas, $\mathrm{K}_{1}$ e da eficiência de adesão, $\eta$, através das Equações (1) e (2), obtidas por balanço de massa no equipamento:

$$
\begin{gathered}
\mathrm{K}_{1}=\frac{\overline{\mathrm{m}}_{\mathrm{p}}-\overline{\mathrm{m}}_{\mathrm{p} 0}}{\theta \cdot \overline{\mathrm{m}}_{\mathrm{p} 0}} \\
\frac{\mathrm{n}_{0}\left(\overline{\mathrm{m}}_{\mathrm{p}} \overline{\mathrm{m}}_{\mathrm{p} 0}\right)}{\mathrm{W}_{\mathrm{s}} \mathrm{C}_{\mathrm{s}}}
\end{gathered}
$$

onde:

$\mathrm{C}_{\mathrm{s}}=$ teor de sólidos na suspensão, [\%];

$\mathrm{K}_{1}=$ taxa de aumento de massa das partículas, $\left[\mathrm{min}^{-1}\right]$; $\overline{\mathrm{m}_{\mathrm{p}}}=$ massa média da cápsula revestida, $[\mathrm{g}] ;$

$\frac{\mathrm{p}}{\mathrm{m}_{\mathrm{p} 0}}=$ massa média inicial da cápsula, [g];

$\mathrm{n}_{0}=$ número inicial de cápsulas no leito, [-];

$\mathrm{W}_{\mathrm{s}}=$ vazão mássica de suspensão de revestimento, [g/

$\min ]$;

$\theta=$ tempo, [min];

$\eta=$ eficiência de adesão, [-];

\section{Ensaios de gastro-resistência}

Ensaios de desintegração foram realizados de forma a verificar as propriedades gastro-resistentes do produto revestido. Foram utilizadas 6 cápsulas, correspondendo a variados intervalos de tempos de revestimento, as quais foram expostas em ácido clorídrico $0,1 \mathrm{~N}$ e mantidas a $37^{\circ} \mathrm{C} \pm 1{ }^{\circ} \mathrm{C}$. Após 60 minutos, as cápsulas foram mergulhadas em solução tampão fosfato $\mathrm{pH}$ 6,8 e mantidas a $37^{\circ} \mathrm{C} \pm 1{ }^{\circ} \mathrm{C}$, até total desintegração. A desintegração neste teste foi considerada completa quando não se observou nenhum resíduo da unidade, à exceção dos fragmentos de revestimento insolúveis ou da matriz. Foram consideradas cápsulas gastro-resistentes, as que permaneceram intactas, com ausência de rachaduras ou amolecimento, no período em que estavam expostas ao fluido gástrico por $60 \mathrm{~min}$ e que se desintegraram em fluido intestinal, em tempo inferior a $45 \mathrm{~min}$. Os ensaios foram realizados segundo o teste de desintegração descrito na Farmacopéia Brasileira (1999).

\section{Ensaios de resistência e deformação}

Os ensaios de resistência mecânica das cápsulas foram realizados por meio de testes de compressão $\mathrm{x}$ deformação até a determinação do ponto onde ocorreu a fratura das cápsulas. Essa compressão foi realizada lateralmente com o auxílio de uma máquina universal (INSTRON 4400). A velocidade de deslocamento das placas de compressão foi de $10 \mathrm{~mm} / \mathrm{min}$ e as análises foram feitas em triplicata.

\section{Microfotografias em Microscópio Eletrônico de Varredura}

O procedimento consistiu primeiramente na fixação das amostras sobre um suporte metálico. A seguir as amostras sofreram um recobrimento metálico com ouro puro. As amostras assim preparadas foram levadas ao microscópio eletrônico de varredura, no qual foram obtidas microfotografias da camada superficial das cápsulas revestidas e não-revestidas. $\mathrm{O}$ microscópio utilizado foi ZEISS, modelo DSM 960.

\section{Variáveis e condições operacionais.}

As variáveis e condições experimentais utilizadas neste estudo são apresentadas na Tabela II. 
TABELA II - Variáveis e faixas de operação utilizadas

\begin{tabular}{lcc}
\hline Variável & Símbolo & Faixa \\
\hline $\begin{array}{l}\text { Razão entre vazão mássica de suspensão de revestimento alimentada pela } \\
\text { vazão de gás introduzida ao sistema (-) }\end{array}$ & $\mathrm{W}_{\mathrm{s}} / \mathrm{W}_{\mathrm{g}}$ & $0,0014-0,0020$ \\
$\begin{array}{l}\text { Razão entre a vazão de ar introduzida pela vazão correspondente à condição } \\
\quad \text { de jorro mínimo (-) }\end{array}$ & $\mathrm{Q}^{/} \mathrm{Q}_{\mathrm{jm}}$ & 2,5 e 3,0 \\
$\begin{array}{l}\text { Massa de cápsulas colocada no equipamento }(\mathrm{g}) \\
\text { Tamanho das cápsulas }(-)\end{array}$ & $\mathrm{M}_{\mathrm{o}}$ & 150,0 e 300,0 \\
Vazão do ar de atomização $(\mathrm{L} / \mathrm{min})$ & $\mathrm{T}_{\text {caps }}$ & 0 e 1 \\
Temperatura de entrada do gás de jorro $\left({ }^{\circ} \mathrm{C}\right)$ & $\mathrm{W}_{\text {at }}$ & 15,0 \\
Temperatura da suspensão de revestimento $\left({ }^{\circ} \mathrm{C}\right)$ & $\mathrm{T}_{\mathrm{g}}$ & $60,0^{\circ} \mathrm{C}$ \\
Concentração de Sólidos na Suspensão de Revestimento $(\%$ de sólidos $)$ & $\mathrm{T}_{\mathrm{s}}$ & Ambiente $\cong 30,0$ \\
\hline
\end{tabular}

\section{RESULTADOS E DISCUSSÃO}

\section{Fluidodinâmica do recobridor de leito de jorro}

As Figuras 2a, 2b, 2c e 2d apresentam, respectivamente, gráficos típicos de queda de pressão no leito em função da vazão de ar introduzida no sistema para as cápsulas de tamanho 0 e 1, para duas massas distintas, 150 e $300 \mathrm{~g}$. A partir dos gráficos obtidos, determinou-se o valor da queda de pressão máxima do leito, $\Delta \mathrm{P}_{\mathrm{m}}$, da queda de pressão de jorro estável, $\Delta \mathrm{P}_{\mathrm{j}}$ e da vazão de ar de mínimo jorro, $\mathrm{Q}_{\mathrm{jm}}$. A Tabela III apresenta os resultados obtidos.

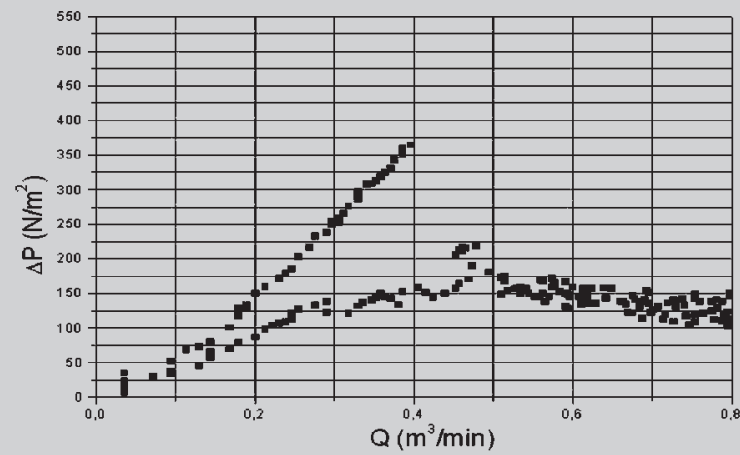

a

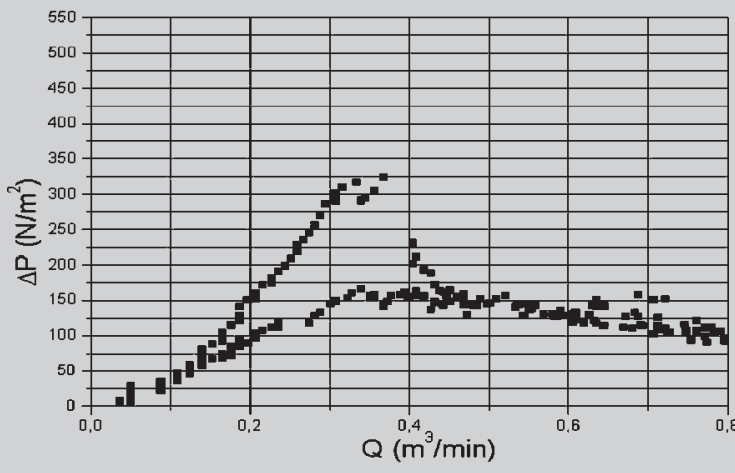

c

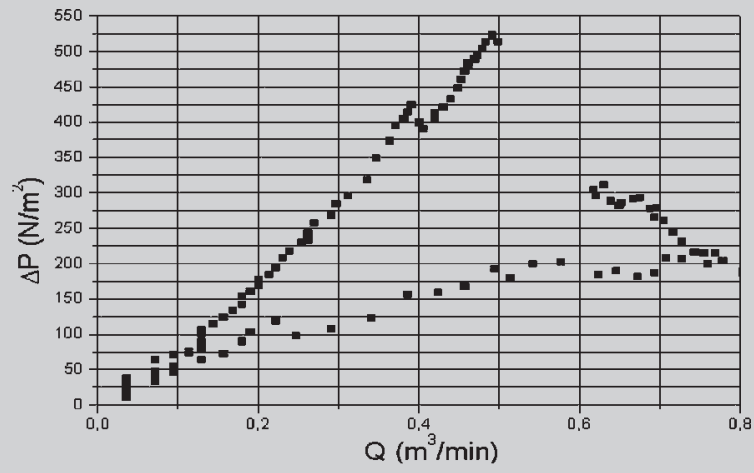

b

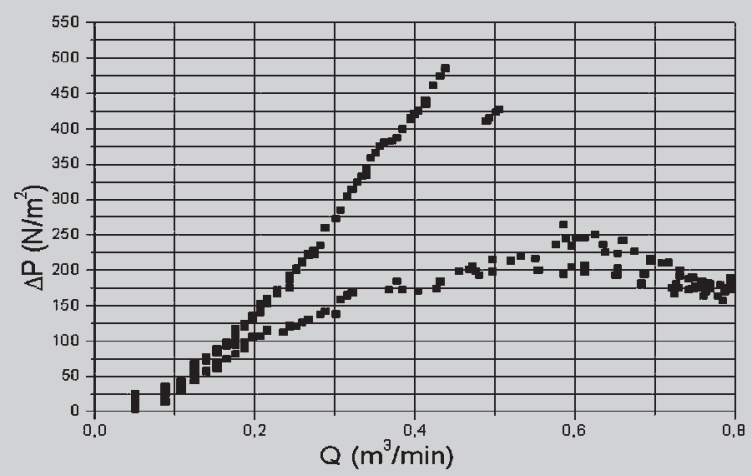

d

FIGURA 2 - Queda de pressão no leito em função da vazão de gás alimentada ao sistema.

$\mathbf{a}, \mathbf{b}-\mathrm{T}_{\text {caps }} 0 ; \mathbf{c}, \mathbf{d}-\mathrm{T}_{\text {caps }} 1 ; \mathbf{a}, \mathbf{c}-\mathrm{M}_{0}=150 \mathrm{~g} ; \mathbf{b}, \mathbf{d}-\mathrm{M}_{0}=300 \mathrm{~g}$. 
O aumento da massa inicial de cápsulas no leito acarretou incremento em todos os parâmetros fluidodinâmicos do equipamento $\left(\mathrm{Q}_{\mathrm{jm}}, \Delta \mathrm{P}_{\mathrm{m}} \Delta \mathrm{P}_{\mathrm{j}}\right)$, como pode ser observado na Tabela III. Este comportamento é esperado, pois com o aumento na massa de partículas no leito, aumenta a altura do leito estático, produzindo conseqüente elevação na resistência ao escoamento do ar, o que reflete em maiores valores de $\Delta \mathrm{P}_{\mathrm{m}}, \Delta \mathrm{P}_{\mathrm{j}}$ e $\mathrm{Q}_{\mathrm{jm}}$.

TABELA III - Parâmetros fluidodinâmicos do leito de jorro para os diferentes tamanhos e massa $\left(\mathrm{M}_{\mathrm{o}}\right)$ de cápsulas utilizadas

\begin{tabular}{ccccc}
\hline $\mathbf{T}_{\text {caps }}$ & $\begin{array}{c}\mathbf{M}_{\mathbf{0}} \\
\mathbf{( g )}\end{array}$ & $\begin{array}{c}\mathbf{\Delta} \mathbf{P}_{\mathbf{m}} \\
\left(\mathbf{N} / \mathbf{m}^{\mathbf{2}}\right)\end{array}$ & $\begin{array}{c}\mathbf{\Delta} \mathbf{P}_{\mathbf{j}} \\
\left(\mathbf{N} / \mathbf{m}^{2}\right)\end{array}$ & $\begin{array}{c}\mathbf{Q}_{\text {jm }} \\
\left(\mathbf{m}^{3} / \mathbf{m i n}\right)\end{array}$ \\
\hline $\mathbf{0}$ & 150 & 370 & 130 & 0,48 \\
$\mathbf{0}$ & 300 & 525 & 210 & 0,73 \\
$\mathbf{1}$ & 150 & 325 & 125 & 0,40 \\
$\mathbf{1}$ & 300 & 485 & 175 & 0,55 \\
\hline
\end{tabular}

\section{Operação de Revestimento}

Amostras de cápsulas, retiradas ao longo da operação de revestimento, permitiram a determinação do aumento de massa da cápsula em função do tempo de processo. A Figura 3 mostra gráfico típico obtido.

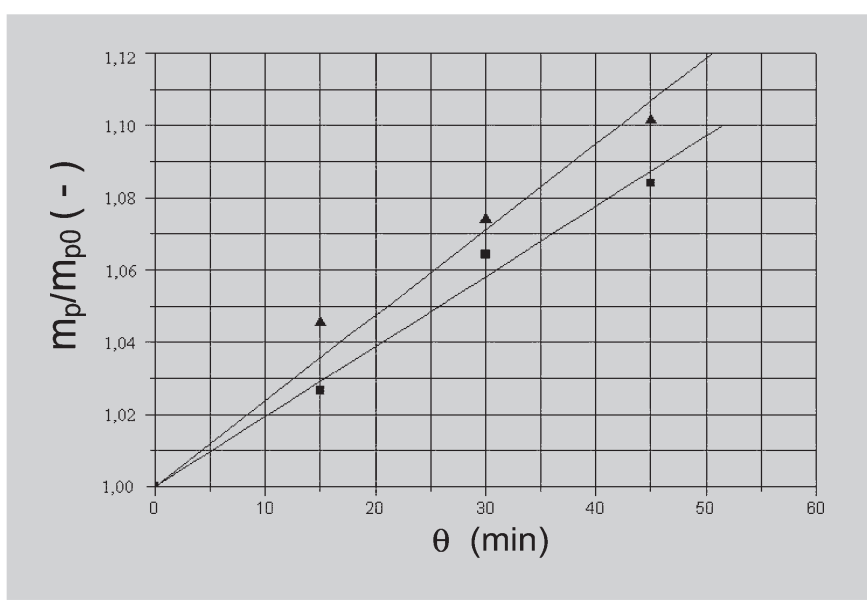

FIGURA 3 - Aumento relativo de massa das cápsulas em função do tempo de revestimento. $\boldsymbol{\Delta}-\mathrm{T}_{\text {caps }} 0 ; \mathbf{\square}-\mathrm{T}_{\text {caps }} 1$

Desse gráfico, pode-se constatar que as massas das cápsulas aumentaram linearmente com o tempo de operação $\left(\mathrm{R}^{2}=0,99\right)$, resultados esses similares aos obtidos em operações de revestimento de comprimidos (Oliveira, 2002). A partir dos resultados de aumento de massa das partículas em função do tempo de revestimento, determinou-se a taxa de aumento de massa das cápsulas $\left(\mathrm{K}_{1}\right)$ e a eficiência de adesão durante a operação $(\eta)$. Esses resultados estão apresentados na Tabela IV, juntamente com as condições experimentais utilizadas.

Observando-se os dados apresentados na Tabela IV,

TABELA IV - Resultados experimentais da taxa de aumento de massa das partículas $\left(\mathrm{K}_{1}\right)$ e da eficiência de adesão ( $)$

\begin{tabular}{ccccccc}
\hline EXP & $\mathbf{T}_{\text {cap }}$ & $\mathbf{M}_{\mathbf{0}}(\mathbf{g})$ & $\mathbf{Q} / \mathbf{Q}_{\mathbf{j m}}(-)$ & $\mathbf{W}_{\mathbf{s}} / \mathbf{W}_{\mathbf{g}}(-)$ & $\mathbf{K}_{\mathbf{1}}\left(\mathbf{m i n}^{-1}\right)$ & $\boldsymbol{\eta} \mathbf{( - )}$ \\
\hline 1 & 0 & 150 & 2,5 & 0,0014 & $0,0024 \pm 0,0001$ & $87,1 \pm 0,14$ \\
2 & 0 & 150 & 2,5 & 0,0020 & $0,0032 \pm 0,0002$ & $82,8 \pm 0,05$ \\
3 & 0 & 150 & 3,0 & 0,0014 & $0,0050 \pm 0,0003$ & $\cong 100,0$ \\
4 & 0 & 150 & 3,0 & 0,0020 & $0,0039 \pm 0,0001$ & $90,9 \pm 0,02$ \\
5 & 0 & 300 & 2,5 & 0,0014 & $0,0023 \pm 0,0002$ & $\cong 100,0$ \\
6 & 0 & 300 & 2,5 & 0,0020 & $0,0034 \pm 0,0003$ & $\cong 100,0$ \\
7 & 0 & 300 & 3,0 & 0,0014 & $0,0023 \pm 0,0001$ & $92,4 \pm 0,03$ \\
8 & 0 & 300 & 3,0 & 0,0020 & $0,0032 \pm 0,0002$ & $87,8 \pm 0,06$ \\
9 & 1 & 150 & 2,5 & 0,0014 & $0,0019 \pm 0,0001$ & $96,8 \pm 0,04$ \\
$10^{*}$ & 1 & 150 & 2,5 & 0,0020 & & - \\
11 & 1 & 150 & 3,0 & 0,0014 & $0,0031 \pm 0,0004$ & $\cong 100,0$ \\
12 & 1 & 150 & 3,0 & 0,0020 & $0,0018 \pm 0,0002$ & $52,5 \pm 0,10$ \\
13 & 1 & 300 & 2,5 & 0,0014 & $0,0010 \pm 0,0001$ & $78,2 \pm 0,10$ \\
14 & 1 & 300 & 2,5 & 0,0020 & $0,0013 \pm 0,0001$ & $64,4 \pm 0,04$ \\
15 & 1 & 300 & 3,0 & 0,0014 & $0,0018 \pm 0,0002$ & $95,5 \pm 0,08$ \\
16 & 1 & 300 & 3,0 & 0,0020 & $0,0020 \pm 0,0001$ & $84,2 \pm 0,08$ \\
\hline
\end{tabular}

* Ocorreu aglomeração das cápsulas no leito. 
pode-se observar que a condição $\mathrm{W}_{\mathrm{s}} / \mathrm{W}_{\mathrm{g}}$ igual a $0,0014 \mathrm{e}$ $\mathrm{Q} / \mathrm{Q}_{\mathrm{jm}}$ igual a 3,0 é uma condição ótima de operação do sistema, apresentando valores de eficiência de adesão superiores a 90\%, para ambas as massas e tamanho de cápsulas estudadas.

\section{Testes de desintegração}

A Figura 4 mostra a relação entre a massa de revestimento aderida por área da cápsula e tempo de desintegração da cápsula. A linha sólida apresentada no gráfico representa o ajuste obtido por regressão não-linear. A seguinte equação foi obtida $(\mathrm{R}=0,99)$.

$$
\theta=2,071+325,52\left(\mathrm{M}_{\mathrm{r}} / \mathrm{A}\right)
$$

A Equação (3) pode ser usada para a determinação do tempo de desintegração da cápsula em qualquer condição experimental, conhecendo-se a massa de revestimento aderida e a área superficial da cápsula. Para razão entre a massa de revestimento aderida e a área superficial da cápsula maior que $0,006 \mathrm{~g} / \mathrm{cm}^{2}$ a cápsula pode ser considerada gastro-resistente, valor este que confirma os resultados descritos em literatura (Avis et al., 1998).

\section{Ensaios de Compressão}

De forma a verificar a relação existente entre a força de compressão e a massa de revestimento depositado

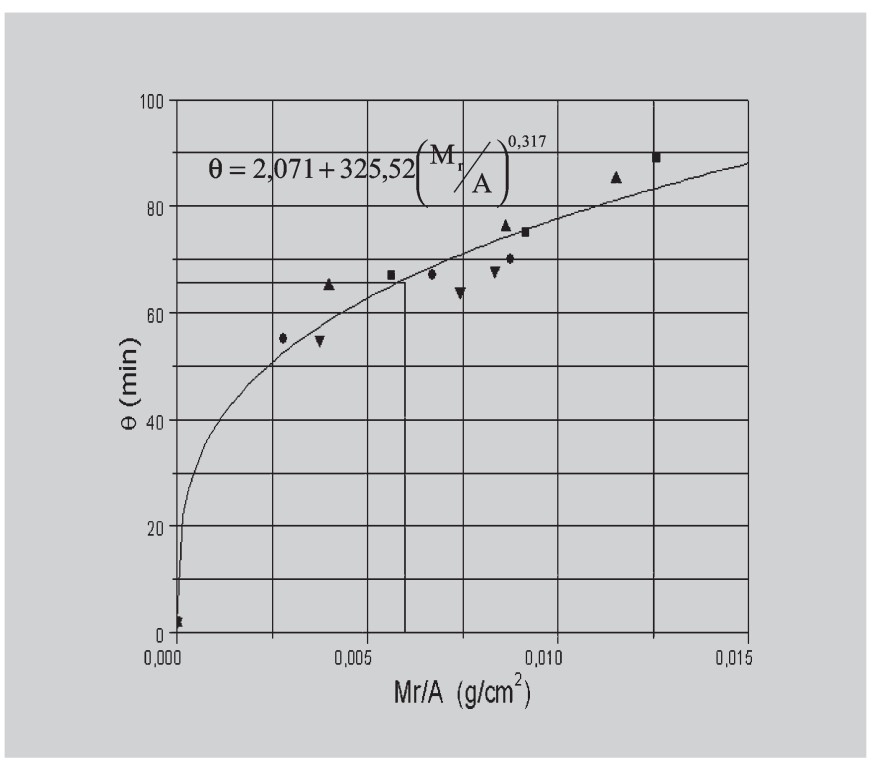

FIGURA 4 - Tempo de desintegração em função da massa de revestimento aderida por área da cápsula. por unidade de área das cápsulas, construiu-se a Figura 5. A linha sólida nessa figura representa ajuste por regressão linear aos dados experimentais. A seguinte equação foi obtida:

$$
\text { FC } 12321,94 \quad \mathrm{M}_{\mathrm{r}} / \mathrm{A} \quad 80,90
$$

A equação (4) apresentou coeficiente de correlação superior a 0,95 e pode ser utilizada na estimativa da resistência da cápsula em função da massa de revestimento aderido por unidade de área da cápsula.

\section{Microfotografias em Microscópio Eletrônico de Varredura}

A Figura 6 mostra micrografias típicas da superfície do revestimento com a ampliação de 10 e 100 vezes, obtidas para as cápsulas duras gelatinosas de tamanho 0 , após 45 minutos de revestimento. A Figura 7 apresenta os mesmos resultados para as cápsulas de tamanho 1. Dessas Figuras, pode-se observar que a camada de revestimento deposita-se uniformemente sobre toda a superfície da cápsula, não sendo constatada a presença de trincas ou rachaduras, o que é uma característica favorável à obtenção do efeito gastro-resistente.

\section{CONCLUSÕES}

As seguintes conclusões podem ser feitas da análi-

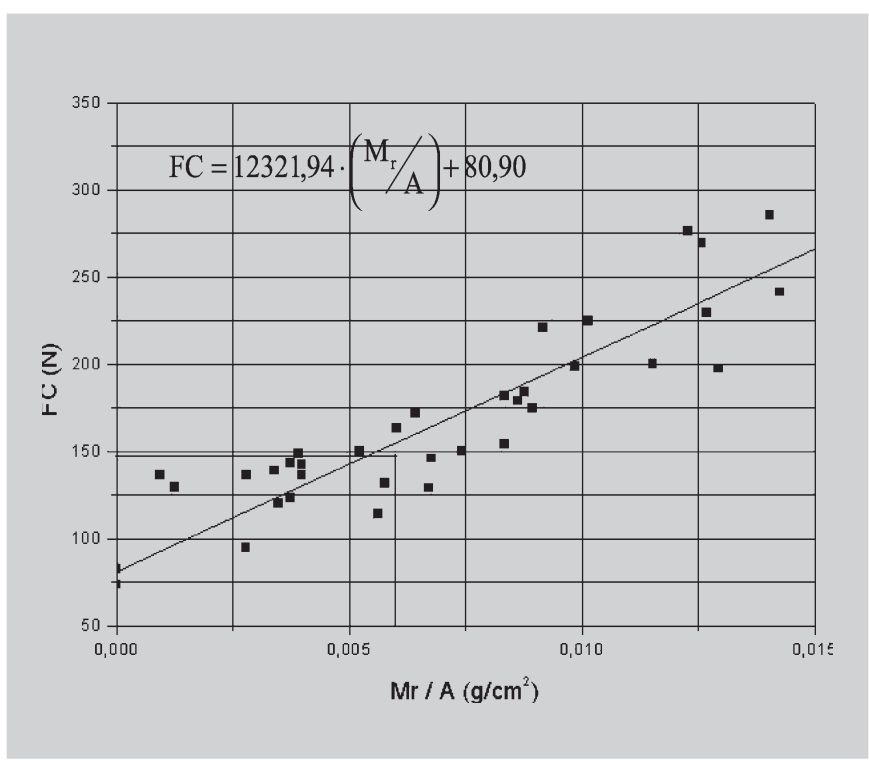

FIGURA 5 - Força de compressão (N) em função da massa de revestimento aderida por área da cápsula $\left(\mathrm{g} / \mathrm{cm}^{2}\right)$. 


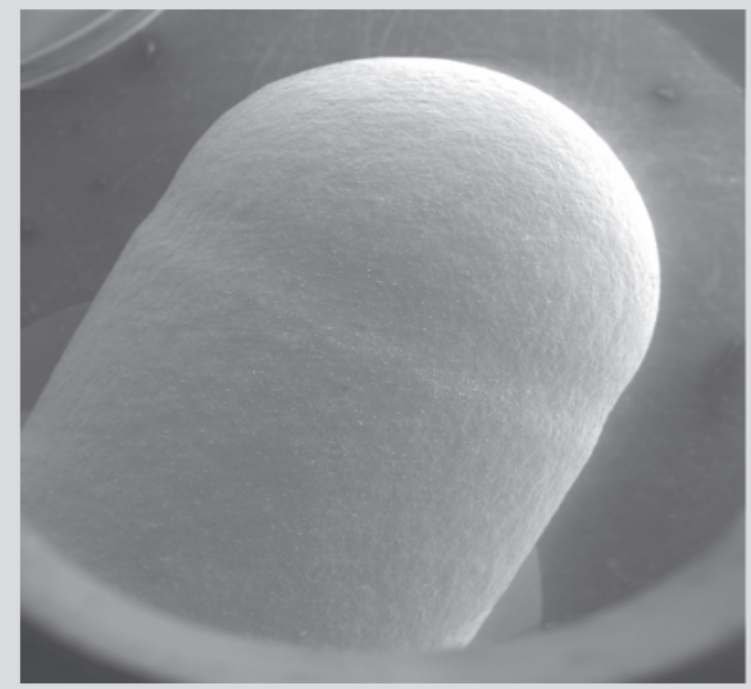

a

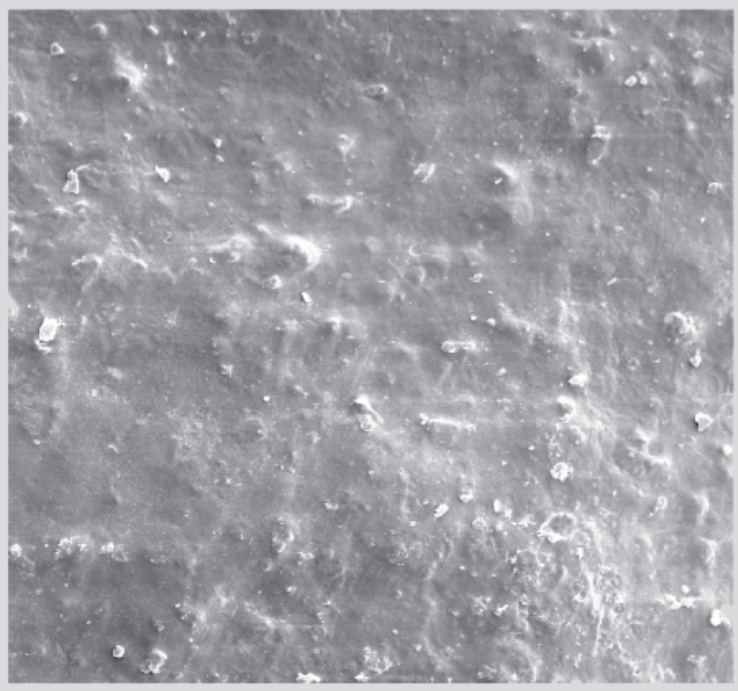

b

FIGURA 6 - Cápsula Dura de Gelatina tamanho 0, 45 min de revestimento. $\mathrm{a}$ - Aumento de $10 \mathrm{x}$ e b-Aumento de $100 \mathrm{x}$.

se dos dados apresentados:

- a massa de revestimento aderida às partículas aumenta linearmente com o tempo de processo;

- altos valores de eficiência de adesão foram obtidos para a maioria das condições experimentais utilizadas nesse estudo, o que mostra a viabilidade do processo em análise para a aplicação de revestimento em cápsulas gelatinosas duras;

- após 30 minutos de aplicação de revestimento todas as cápsulas apresentaram propriedades gastro-resistente, dependente da massa de revestimento depositado por unidade de área da cápsula;

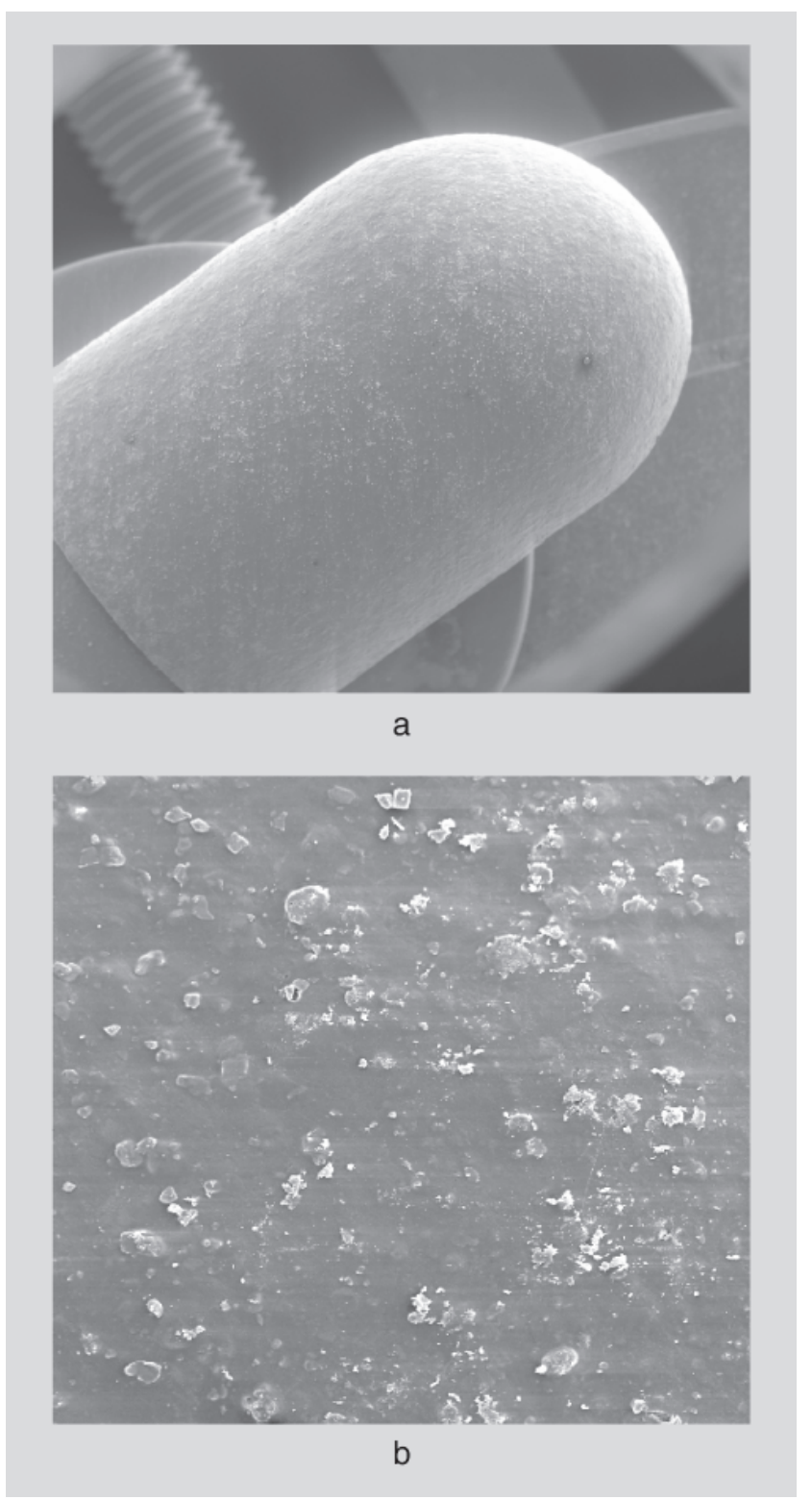

FIGURA 7 - Cápsula Dura de Gelatina tamanho 1, 45 min de revestimento. a - Aumento de $10 \mathrm{x}$ e b-Aumento de $100 \mathrm{x}$.

- o tempo decorrido no ensaio de gastro-resistência em função da massa de revestimento depositada por unidade de área da cápsula pode ser estimado pela equação 3 ;

- a resistência à compressão das cápsulas aumenta proporcionalmente à espessura da camada de revestimento aderida;

- a resistência à compressão das cápsulas revestidas em função da massa de revestimento depositada por toda superfície da cápsula pode ser estimada pela equação 4;

- o revestimento deposita-se uniformemente sobre a superfície da cápsula, não sendo observada a presença 
de defeitos ou rachaduras, sendo esse um fator importante ao desenvolvimento de revestimentos com propriedades gastro-resistentes ou de liberação prolongada;

- para perfeita aplicação do revestimento necessita-se de perfeita junção entre a base e a capa das cápsulas, caso contrário ocorre revestimento irregular nessa região;

- nas condições investigadas a condição indicada para a aplicação do revestimento foi $\mathrm{Q} / \mathrm{Q}_{\mathrm{jm}}$ igual a 3,0 e W/ $\mathrm{W}_{\mathrm{g}}$ igual a 0,0014 .

\section{NOTAÇÃO}

A = área superficial da cápsula, $\left[\mathrm{cm}^{2}\right]$

$\mathrm{C}_{\mathrm{s}} \quad=$ teor de sólidos na suspensão, [\%];

$\mathrm{d}_{\mathrm{p}}=$ diâmetro volumétrico, $[\mathrm{cm}]$;

$\mathrm{EXP}=$ número do experimento, $[-]$;

$\mathrm{FC}$ = força de compressão; [N];

$\mathrm{K}_{1} \quad$ = taxa de aumento de massa das partículas, [1/min];

$\overline{\mathrm{m}_{\mathrm{p}}}=$ massa da cápsula revestida, $[\mathrm{g}]$;

$\overline{\mathrm{m}}_{\mathrm{p} 0}=$ massa inicial da cápsula, [g];

$\mathrm{M}_{0}=$ massa inicial de cápsula no leito, $[\mathrm{g}]$;

$\mathrm{M}_{\mathrm{r}}=$ massa de revestimento aderida, [g];

$\mathrm{n}_{0}=$ número inicial de cápsulas no leito, [-];

$\mathrm{P}_{\text {at }}=$ pressão do ar de atomização, $\left[\mathrm{kgf} / \mathrm{cm}^{2}\right]$;

$\mathrm{Q}=$ vazão de $\mathrm{ar},\left[\mathrm{m}^{3} / \mathrm{min}\right]$;

$\mathrm{Q}_{\mathrm{jm}}=$ vazão de ar de jorro mínimo, $\left[\mathrm{m}^{3} / \mathrm{min}\right]$;

$\mathrm{Se}=$ superfície específica do material inerte; $\left[\mathrm{cm}^{2} / \mathrm{g}\right]$;

$\mathrm{T}_{\text {caps }}=$ tamanho da cápsula

$\mathrm{T}_{\mathrm{g}}^{\text {caps }}=$ temperatura do ar de jorro, $\left[{ }^{\circ} \mathrm{C}\right]$;

$\mathrm{T}_{\mathrm{s}}^{\mathrm{g}}=$ temperatura da suspensão de revestimento, $\left[{ }^{\circ} \mathrm{C}\right]$;

$\mathrm{W}_{\mathrm{at}}$ = vazão de ar de atomização, [L/min];

$\mathrm{W}_{\mathrm{g}}^{\text {at }}=$ vazão de ar introduzida no sistema, $\left[\mathrm{m}^{3} / \mathrm{min}\right]$;

$\mathrm{W}_{\mathrm{s}}^{\mathrm{g}}=$ vazão mássica de suspensão de revestimento, [g/min];

$\theta \quad=$ tempo, [min];

$\phi \quad=$ fator de forma, [ - ];

$\gamma \quad=$ ângulo da base cônica; [graus];

$\eta \quad=$ eficiência de adesão, [ - ];

$\Delta \mathrm{P}_{\mathrm{m}}=$ queda de pressão máxima, $\left[\mathrm{N} / \mathrm{m}^{2}\right] ;$

$\Delta \mathrm{P}_{\mathrm{j}}=$ queda de pressão de jorro estável, $\left[\mathrm{N} / \mathrm{m}^{2}\right]$;

\section{ABSTRACT}

\section{Utilization of a spouted bed for the application of enteric coating in hard gelatin capsules}

In this work is presented a study of the viability of the use of the spouted bed for application of the gastric resistant coating on hard gelatin capsules. The parameters studied were the ratio between the mass feed flow rate of the coating suspension to the mass feed flow rate of the gas introduced to the system, $\left(W_{s} / W_{g}\right)$, the ratio between the flow rate of the spouting gas relative to the minimum spouting, $\left(Q / Q_{j m}\right)$, the mass of capsules loaded to the equipment, $\left(M_{0}\right)$ and the capsule's size, $\left(T_{\text {caps }}\right)$. The performance of the process as well as the coating quality were evaluated through the increase rate of the particle mass, $\left(K_{\nu}\right)$, by the adhesion efficiency, $(\eta)$, disintegration and compression tests and by photomicrographs obtained in a scanning electronic microscope. The experiments were carried out in a spouted bed with $15 \mathrm{~cm}$ of the cylindrical column and with an included angle of the conical base of the $40^{\circ}$. The results lead to the determination of the best condition for application of the coating on hard gelatin capsules: $W_{s} / W_{g}=0.0014$ and $Q / Q_{j m}=$ 3.0, for both mass of capsules loaded to the system and capsule's size investigated. The disintegration tests showed that the gastric resistant properties are obtained with a coating mass higher than $0.006 \mathrm{~g} / \mathrm{cm}^{2}$.

UNITERMS: Spouted bed. Enteric coating. Eudragit L30-D-55. Hard gelatin capsules.

\section{AGRADECIMENTOS}

Os autores agradecem à Fundação de Amparo à Pesquisa do Estado de São Paulo (FAPESP), pelo suporte financeiro, à ALMAPAL S.A. e à CAPSUGEL, pela doação do Eudragit L-30-D-55 e das cápsulas gelatinosas duras, respectivamente.

\section{REFERÊNCIAS BIBLIOGRÁFICAS}

AVIS, K. E.; SHUKLA, A. J.; CHANG, R.-K., eds. Pharmaceutical unit operations: Coating. Buffalo Grove: Interpharm Press, 1999. 348p. (Drug manufacturing technology series, v.3).

BODMEIER, R. Coating of solid dosage forms. Eur. $J$. Pharm. Biopharm., Shannon, v.47, p.1., 1999.

COLE, E. T.; SCOTT, R. A.; CONNOR, A. L.; WILDING, I. R.; PETEREIT, H. U.; SCHMINKE, C.; BECKERT, T.; CADE, D. Enteric coated HPMC capsules designed to achieve intestinal targeting. Int. J. Pharm., Amsterdam, v.231, n.1, p.83-95, 2002.

DOANE, W.M.; SHASKA, B.S.; RUSSELL, C.R. Encapsulation of pesticides within starch matrix in controlled release pesticides. Washington: ACS, 1977. p.16-16. (ACS Symposium Series, v.173). 
FARMACOPÉIA Brasileira. 4.ed. São Paulo: Atheneu, 1988. pt. 1. v.1.4.1-v.1.4.2-2.

FELTON, L.A.; SHAH, N.H.; ZHANG, G.; INFELD, M.H.; MALICK, A.W.; MCGINITY, J.W. Physicalmechanical properties of film-coated soft gelatin capsules. Int. J. Pharm., Amsterdam, v.127, n.2, p.203211, 1996.

FELTON, L.A.; HAASE, M.M.; SHAH, N.H.; ZHANG, G.; INFELD, M.H.; MALICK, A.W.; MCGINITY, J.W. Physical and enteric properties of soft gelatin capsules coated with Eudragit L-30-D-55. Int. J. Pharm., Amsterdam, v.113, n.1, p.17-24, 1995.

KALALA, W.; KINGET, R.; VANDENMOOTER, G.; SAMYN, C. Colonic drug-targeting: in vitro release of ibuprofen from capsules coated with poly(ether-ester) azopolymers. Int. J. Pharm., Amsterdam, v.139, n.1/2, p.187-195, 1996.

KUCHARSKI, J.; KMIEC, A. Hydrodynamics, heat and mass transfer during coating of tablets in a spouted bed. Can. J. Chem. Eng., Ottawa, v.61, n.3, p.435-439, 1983.

MATHUR, K.B.; EPSTEIN, N. Dynamics of spouted beds. Adv. Chem. Eng., San Diego, v.9, p.111-191, 1974.

OLIVEIRA, W.P. Contribuição ao estudo do processo de revestimento de partículas em leito de jorro. Ribeirão Preto, 2002. 139p. (Tese de Livre Docência - Faculdade de Ciências Farmacêuticas de Ribeirão Preto Universidade de São Paulo).
PICCININI, N.; ROVERO, G. Thick coatings by thermochemical deposition in spouted bed. Can. J. Chem. Eng., Ottawa, v.61, p.448-453, 1983.

PINA, M. E.; SOUSA, A. T.; BROJO, A. P. Coating of hard gelatin capsules. 1. Application of hydroalcoholic solutions of formaldehyde in preparation of gastroresistant capsules. Int. J. Pharm., Amsterdam, v.133, n.1/ 2, p.139-148, 1996.

PINA, M. E.; SOUSA, A. T.; BROJO, A. P. Enteric coating of hard gelatin capsules. 2. Lioavailability of formaldehyde treated capsules. Int. J. Pharm., Amsterdam, v.148, n.1, p.73-84, 1997.

PORTER, E. F. Coat seeds with polymers. CHEMTECH, Washington, v.8, n.5, p.284-287, 1978.

SALMAN, O. A. Polyethylene-coated urea. 1. Improves storage and handling properties. Ind. Eng. Chem. Res., Columbus, v.28, p.630-632, 1989.

SALMAN, O. A.; HOVAKEEMIAN, G.; KHRAISHI, N. Polyethylene-coated urea 2 . Urea release as affected by coating material, soil type and temperature. Ind. Eng. Chem. Res., Columbus, v.28, p.633-638, 1989.

Recebido para publicação em 05 de abril de 2003. 